\title{
INFORMES $^{1}$
}

\section{La información y atención al ciudadano y la tramitación de prodecimientos administrativos por medios electrónicos (Internet) ${ }^{2}$}

\section{INTRODUCCIÓN}

En números anteriores de esta Revista y en esta misma Sección hemos comentado los Decretos 57/1983, de 9 de marzo, 260/1988 y 262/1988, ambos de 2 de agosto y los Reales Decretos 208/1996, de 9 de febrero y 772/1999, de 7 de mayo. Estas normas han regulado las Oficinas de Información de la Comunidad Autónoma, la racionalización administrativa de la Junta de Andalucía, el Libro de Sugerencias y Reclamaciones (vid. el número 3 de la Revista), los servicios de información administrativa y atención al ciudadano (vid. el número 28 de la Revista) y la presentación de escritos, expedición de copias y régimen de las oficinas de registro (vid. el número 34 de la Revista). En esta ocasión, enlazando con las disposiciones citadas y más cercanamente con el Decreto 72/2003, de 18 de marzo, de Medidas de Impulso de la Sociedad del Conocimiento en Andalucía (vid. esta Sección en el número 49 de la Revista), nos corresponde dar noticia del Decreto 183/2003, de 24 de junio, por el que se regula la información y atención al ciudadano y la tramitación de procedimientos administrativos por medios electrónicos (Internet), publicado en el BOJA número 134, de 15 de julio, y en vigor desde el 15 de septiembre.

Con este nuevo reglamento se da un paso adelante para la satisfacción del reto asumido por Andalucía de convertirse en una de las Comunidades más avanzadas de Europa, como pone de manifiesto la Exposición de Motivos. Se inserta la disposición, pues, en el entorno que diseña el Decreto 72/2003, antes citado, al que paradójicamente ni menciona ${ }^{3}$.

\footnotetext{
${ }^{1}$ Sección redactada bajo la dirección de José Ignacio Morillo-Velarde Pérez.

${ }^{2}$ Realizado por Mํa del Carmen Núñez Lozano.

3 Además de la lógica referencia al portal "www.andaluciajunta.es", ambos comparten la cita en las Exposiciones de Motivos del Plan de Iniciativas Estratégicas para el Desarrollo de la
} 
El Decreto tiene por objeto, según señala el art. 2.1, regular la prestación de servicios de información y de atención al ciudadano por los órganos, unidades y entidades a los que se aplica y que inmediatamente veremos, así como la tramitación de procedimientos administrativos a través de redes abiertas de telecomunicación, incluyéndose las relaciones con las demás Administraciones y entidades públicas cuando actúen como usuarios de los servicios o como interesados en los procedimientos a los que resulte de aplicación este Decreto. Asimismo, regula la utilización de medios y técnicas electrónicas e informáticas relacionados con su objeto, en particular el Registro telemático y los sistemas de acreditación y firma electrónica que permitan garantizar la autenticidad, seguridad y confidencialidad de las relaciones a las que se refiere la disposición.

El apartado segundo del art. 2 señala que también es su objeto la regulación de las relaciones internas entre los órganos, unidades y entidades, incluidos los que se realicen a través de redes internas (intranet). Ello -especifica a continuación- sin perjuicio de las relaciones interadministrativas por medios electrónicos, informáticos o telemáticos que puedan institucionalizarse a través de los oportunos Convenios. Sin embargo, el Decreto no llega a disciplinar tales relaciones, o al menos no lo hace de modo directo, pues lo único que contiene son previsiones dispersas.

La disposición se aplica a todos los órganos y unidades de la Administración de la Junta de Andalucía y sus organismos autónomos, así como, en cuanto ejerciten potestades administrativas, a los consorcios en los que sea mayoritaria la representación, directa o indirecta, de la Administración de la Junta de Andalucía (art. 1.1); también se aplica a las demás entidades vinculadas o dependientes de la Administración de la Junta de Andalucía, contempladas en los artículos 6 y 6 bis de la Ley 5/1983, de 19 de julio, General de la Hacienda Pública de la Comunidad Autónoma de Andalucía, en tanto ejerciten potestades administrativas ${ }^{4}$ (art. 1.2).

Sociedad de la Información en Andalucía y del Plan Director de Organización para la Calidad de los Servicios. Uno y otro Decreto proceden de Consejerías distintas, el 72 de la de Presidencia y el que ahora se analiza de la de Justicia y Administración Pública, lo que puede explicar el que en el segundo se ignore al primero.

\footnotetext{
${ }^{4}$ Esto es, a las sociedades mercantiles en cuyo capital sea mayoritaria la participación, directa o indirecta, de la Administración de la Junta de Andalucía o de sus Organismos Autónomos y demás entidades de derecho público, a las entidades de derecho público con personalidad jurídica que por Ley hayan de ajustar sus actividades al ordenamiento jurídico privado, a los consorcios, fundaciones y demás entidades con personalidad jurídica propia en los que sea mayoritaria la representación directa o indirecta de la Administración de la Junta de Andalucía.
} 
Está previsto que se pueda aplicar a las Universidades públicas y Entidades Locales de Andalucía en la medida que determinen los Convenios de colaboración que para estos fines puedan suscribir con la Consejería competente; igualmente se indica que este tipo de Convenio se puede suscribir con el Parlamento de Andalucía, el Defensor del Pueblo y la Cámara de Cuentas de Andalucía (Disposición adicional primera). Realmente, sólo tiene sentido esta aplicación del Decreto en virtud de convenio en lo que concierne al Registro telemático del que después nos ocuparemos, esto es, a los efectos de utilizarlo en las relaciones con las Instituciones que se mencionan $^{5}$.

Las materias que regula el Decreto se incardinan fundamentalmente, al decir de la Exposición de Motivos, en el concepto de procedimiento administrativo común. No obstante, la Comunidad Autónoma esgrime como títulos idóneos para amparar la competencia que con la disposición ejercita el art.15.1.1 (régimen jurídico) y el art. 13.4 (procedimiento administrativo derivado de las especialidades de su organización), ambos del Estatuto de Autonomía. También se invoca el art. 45 de la Ley 30/1992, a cuyo tenor las "Administraciones Públicas impulsarán el empleo y aplicación de las técnicas y medios electrónicos, informáticos y telemáticos, para el desarrollo de su actividad y el ejercicio de sus competencias ..."

El Decreto se estructura en seis Capítulos, a los que siguen cinco Disposiciones adicionales, tres transitorias, la Disposición derogatoria ${ }^{7}$ y tres disposiciones finales; cuenta además con tres Anexos. El primer Capítulo contiene las denominadas "Disposiciones generales", que se refieren al ámbito de aplicación, objeto, definiciones, garantías generales de carácter sustantivo y principios de funcionamiento. El segundo se dedica a los servicios de información y atención al ciudadano a través de medios electrónicos (Internet). El tercero al registro y archivo de documentos electrónicos. El cuarto a los requi-

\footnotetext{
${ }^{5}$ Parece claro que cualquier Universidad puede poner a disposición de los ciudadanos información a través de redes abierta de telecomunicación sin necesidad de suscribir un convenio con la Junta de Andalucía.

${ }^{6}$ La Exposición de Motivos puntualiza que "sin otro límite que el de respetar, como es lógico, el propio régimen sustantivo de las instituciones y figuras del procedimiento que sean objeto de dinamización por dichos medios".

${ }^{7}$ Deroga expresamente el art. 28 del Decreto 204/1995, de 29 de agosto, por el que se establecen medidas organizativas para los servicios administrativos de atención directa a los ciudadanos.
} 
sitos y eficacia de los documentos y comunicaciones electrónicas. El quinto a los procedimientos administrativos electrónicos. El sexto, finalmente, a las aplicaciones, programas, medios y soportes. Veamos a continuación algunos de los aspectos más importantes o llamativos de la nueva regulación.

\section{GARANTÍAS GENERALES DE CARÁCTER SUSTANTIVO Y PRINCIPIOS DE FUNCIONAMIENTO}

Las garantías generales de carácter sustantivo se establecen en el art. 4. El apartado primero se remite a la legislación que regula el tratamiento automatizado de la información, la protección de datos, derechos sobre la propiedad intelectual y servicios de la sociedad de la información. El apartado segundo especifica que los datos de carácter personal que la Administración obtenga por el solo hecho de que los ciudadanos realicen consultas o reciban información a través de redes abiertas de telecomunicación no podrán formar parte de un fichero o base de datos administrativa, salvo autorización expresa del interesado, sin perjuicio de que se puedan utilizar datos que no tengan carácter personal con fines estadísticos.

Los principios que rigen las relaciones a través de redes abiertas de telecomunicación son los de simplificación y agilización de trámites, gratuidad, libre acceso, confidencialidad y de seguridad y autenticidad en orden a la identificación de las partes y el objeto de la comunicación (art. 5.1).

En particular, llama la atención el principio de simplificación, tan en la vanguardia desde hace ya varios años. El Decreto señala que las Consejerías, organismos y entidades competentes para la gestión de procedimientos administrativos deberán promover la aplicación del principio en la presentación de escritos y documentos y en la tramitación de los expedientes que se realicen a través de redes abiertas de telecomunicación (art. 5.4). Más adelante se prescribe que las Órdenes de las respectivas Consejerías mediante las cuales se establezca la tramitación telemática de los distintos procedimientos arbitrarán los medios necesarios para avanzar en la reducción y simplificación de su tramitación. En concreto, han de evitar a los interesados la aportación de documentos que obren en poder de la Administración de la Junta de Andalucía o de otras Administraciones Públicas; al efecto, pueden prever la obtención de datos de los órganos administrativos en que se hallen previo consentimiento del interesado, suscribiendo los convenios necesarios con las demás Administraciones para el acceso y consulta a sus bases de datos. Igualmente han de propiciar la sustitución de la aportación de documentos 
acreditativos del cumplimiento de requisitos por una declaración responsable del interesado que exprese la concurrencia de dichos requisitos y el compromiso de aportar los justificantes a requerimiento de la Administración, todo ello en la medida en que lo permitan las disposiciones reguladoras de los respectivos procedimientos (art. 16.2).

Como se aprecia, y aunque el Decreto no lo mencione expresamente, estas previsiones son igualmente de aplicación en los procedimientos tramitados de modo convencional. En cualquier caso, se echa de menos una mayor concreción de estos aspectos, que hubieran podido ser desarrollados con amplitud semejante a la que dedican los arts. 14 y 15 del RD 262/1996 a los certificados telemáticos y a las transmisiones de datos.

Respecto del principio de gratuidad -ausente en la normativa estatal- se matiza que no excluye la posible exigencia de tasas o precios públicos por la prestación de servicios o la tramitación de procedimientos a través de redes abiertas de telecomunicación siempre que así se contemple en normas que resulten de aplicación, ni tampoco la posibilidad de explotar a través de dichas redes documentos electrónicos o bases de datos generados por los órganos y entidades a que se refiere el art. 1 en el ejercicio de sus competencias (art. 5.2).

De otro lado, la regla de la accesibilidad queda restringida, como es natural, en los supuestos de peticiones de información o de documentación que no haya sido previamente puestas a disposición del público en la red en aquellos casos en que la divulgación de un documento o información pueda afectar a la seguridad pública, al honor, la intimidad y seguridad de las personas de acuerdo con la legislación aplicable en materia de archivos, bases de datos públicas y protección de datos personales (art. 5.3).

\section{LOS SERVICIOS DE INFORMACIÓN Y DE ATENCIÓN AL CIUDADANO A TRAVÉS DE MEDIOS ELECTRÓNICOS}

La información y documentación que ha de estar a disposición del público a través de redes abiertas de telecomunicación, para cuyo acceso no es necesaria la firma electrónica, es muy amplia. Debe comprender, como mínimo, la relativa a la organización, competencias y actividades de las Consejerías, organismos y entidades de la Administración de la Junta de Andalucía; las guías de funciones y cartas de servicios que se aprueben en el ámbito de las competencias de dichos organismos y entidades; la normativa que se publique en el BOJA, con la posibilidad de su descarga a través de la 
red; y la información de interés general que se considere oportuna, ya sea de carácter ocasional o permanente, referida ésta a determinados sectores de la actividad administrativa de especial incidencia social en el ámbito de la sanidad, la educación o la cultura. Igualmente, ha de comprender la relativa a los procedimientos administrativos que se tramitan por la Administración de la Junta, con indicación del plazo de resolución y del sentido del silencio; los principales formularios que en los mismos sean de aplicación y de cuáles de esos procedimientos, y qué fases de los mismos, pueden tramitarse a través de la red; así como la información sobre contratación administrativa en el ámbito de la Administración de la Junta de Andalucía, con inclusión de los pliegos de cláusulas administrativas y técnicas, generales y particulares, y de los anuncios de licitación que se produzcan (art. 7.2) ${ }^{8}$. También está prevista la atención de peticiones concretas de documentación o información a través de la red, en los casos en que no hayan sido previamente puestas a disposición del público (art. 8.1) ${ }^{9}$.

La formulación de reclamaciones y sugerencias se sigue rigiendo, en cuanto a su tramitación, por el Decreto 262/1988 y demás normativa aplicable, si bien se advierte que la presentación de sugerencias (y también de peticiones concretas de documentos o de información) puede requerir de alguna modalidad de firma electrónica, lo que en todo caso se exige para la presentación de reclamaciones (art. 8.2 y 3).

\section{REGISTRO Y ARCHIVO DE DOCUMENTOS ELECTRÓNICOS}

Los arts 9 a 11 se ocupan del Registro telemático, archivo de documentos electrónicos y acceso a los mismos y copias. En particular, interesa lo concerniente al Registro y al acceso.

\footnotetext{
${ }^{8}$ A la vista de esta enumeración, llama la atención el art. 7.1, que precisa que la información y documentación puesta a disposición del público a través de las redes abiertas de telecomunicación, para cuyo acceso no sea preciso utilizar ninguna modalidad de firma electrónica, no implica sustitución ni detrimento de la atención personalizada a prestar en las oficinas públicas o por cualquier otro medio. Lo cierto es que parece más completa la información que se obtiene a través de Internet que la que puedan suministrar de modo instantáneo en las oficinas públicas; por otro lado, mientras que la consulta en la red consume poco tiempo, la atención física o a través de otro medio conlleva normalmente mucha más demora.

${ }^{9}$ El Anexo II especifica puntualmente los servicios de información y atención al ciudadano que desde el día dieciséis de septiembre deben estar disponibles en red, sin perjuicio de los que ya lo estuvieran con anterioridad (vid. la Disposición adicional quinta).
} 
El Decreto crea un Registro telemático único, habilitado sólo para la recepción o transmisión de documentos electrónicos relativos a las actividades, servicios o procedimientos que contempla la norma (art. 9.1). La Disposición adicional segunda, apartado segundo, precisa que el Registro sólo funcionará para los procedimientos administrativos que sean competencia directa de las Consejerías y Organismos Autónomos de la Junta de Andalucía; se exceptúan los procedimientos tributarios competencia de la Consejería de Economía y Hacienda, que corresponden a un Registro telemático tributario dependiente de la Consejería de Economía y Hacienda que el mismo Decreto crea, si bien su regulación, entrada en funcionamiento y aplicación se ha de efectuar mediante Orden de la Consejería (Disposición adicional tercera). La tramitación de procedimientos a través del Registro telemático único -que como vemos no es realmente único- por los consorcios y demás entidades que menciona el art. 1 ha de disponerse por Orden de la Consejería correspondiente (segundo párrafo del segundo apartado de la Disposición adicional segunda). Como ya se dijo, la utilización del Registro por otras instituciones requiere de un convenio previo $^{10}$.

En consonancia con el art. 38.9 de la Ley 30/1992, el Registro permitirá la entrada de documentos todos los días del año durante las veinticuatro horas. La fecha de entrada y salida se acreditará mediante un servicio de consignación electrónica de fecha y hora; a efectos del cómputo de dichos términos y plazos, se entenderá que la recepción de documentos electrónicos en un día inhábil en el ámbito territorial del órgano competente para tramitarlo surtirá sus efectos el primer día hábil siguiente, siendo ésta la fecha en la que el documento ha tenido entrada en el Registro del órgano competente para su tramitación (art. 9.3) ${ }^{11}$. El Registro telemático emite automáticamente

\footnotetext{
${ }^{10}$ Ha de tenerse en cuenta que el art. 38.9 de la Ley 30/1992 ciñe los registros telemáticos a los procedimientos y trámites de la competencia del órgano o entidad que los crea. Ha de concluirse, en consecuencia, que el Decreto que comentamos opera con un concepto muy amplio de Administración Pública, en el que incluye a las entidades de los arts. 6 y 6 bis de la Ley General de la Hacienda Pública de la Comunidad (más generosamente, pues, que el RD 263/1996, de 16 de febrero, por el que se regula la utilización de técnicas electrónicas, informáticas y telemáticas por la Administración General del Estado). Más dificultad presenta la adecuación de la Disposición adicional primera al art. 38.9 citado, aunque ha de reconocerse que responde al mandato de eficacia del art. $103 \mathrm{CE}$.
}

${ }^{11}$ El Decreto presupone la inmediatez de la llegada del documento al Registro del órgano competente para tramitarlo y por ello identifica la fecha de recepción en el Registro telemático (fijada en el primer día hábil) con la fecha de recepción en el Registro del órgano competente, obviando la distinción de fechas del art. 4 del RD 772/1999. 
un justificante de la recepción de los documentos en el que se da constancia del asiento de entrada asignado; el justificante se hace llegar a su destinatario en el momento inmediatamente posterior al que tenga lugar el asiento del documento recibido (art. 9.5). Entró en funcionamiento a las cero horas del día 16 de septiembre.

En cuanto al acceso, especifica que el derecho conlleva el de obtener copias o certificados de los documentos cuyo examen sea autorizado por órgano, organismo o entidad competente, previo pago, en su caso, de las exacciones legalmente establecidas. La emisión de copias y certificados en forma de documentos o soportes electrónicos debe contar, para su validez, con la firma electrónica avanzada del titular del órgano que la expide (art. 10.2).

\section{REQUISITOS Y EFICACIA DE LOS DOCUMENTOS Y COMUNI- CACIONES ELECTRÓNICAS}

El Decreto regula con detalle los requisitos del sistema para la transmisión y recepción de información en red o de documentos electrónicos. Las exigencias son mayores en función de la naturaleza de la relación que se establezca o del uso que se vaya a dar a los medios, documentos y soportes electrónicos. El nivel mayor es el de uso en las relaciones jurídico-administrativas que contempla el Decreto, en particular las comunicaciones entre el interesado y los órganos, unidades y entidades que conforman el ámbito de aplicación de la norma que se deban producir en la tramitación de procedimientos administrativos; para estos casos es necesario, además de los restantes requisitos que especifican los apartados 1 y 2 del art. 12, que el destinatario y el remitente utilicen una firma electrónica avanzada basada en un certificado reconocido y producida por un dispositivo seguro de creación de firma ${ }^{12}$, y que dicha firma se acompañe de un dispositivo o servicio de consignación de fecha y hora que permita acreditar el momento exacto en que la comunicación se produce y que, a su vez, permita evitar el rechazo de dicha comunicación por el remitente o el destinatario (art. 12.3).

De otro lado, los documentos emitidos por los órganos, organismos o entidades incluidas en el ámbito de aplicación del Decreto son válidos siempre

\footnotetext{
12 El art. 13 regula la firma electrónica avanzada. Los servicios de certificación y expedición de firmas electrónicas avanzadas los pueden prestar la Fabrica Nacional de Moneda y Timbre-Real Casa de la Moneda, los proveedores habilitados de conformidad con el Real Decreto-Ley 14/1999, de 17 de septiembre, y las entidades que conforme al apartado 2 del art. 13 se acrediten al efecto.
} 
que se garantice su autenticidad, integridad, conservación y demás garantías y requisitos exigidos por la normativa aplicable. En las mismas condiciones son válidas las copias de documentos originales almacenados por medios electrónicos o informáticos (art. 14, trasunto del art. 45.5 de la Ley 30/1992) ${ }^{13}$.

Se regulan también las notificaciones mediante medios electrónicos o informáticos, reiterándose lo que dispone el art. 59.3 de la Ley 30/1992. Es necesario que el interesado haya señalado o consentido expresamente este medio de notificación mediante la identificación de una dirección electrónica al efecto; tal consentimiento puede producirse y revocarse en cualquier momento del procedimiento (art. 15.1) ${ }^{14}$. La notificación se entiende practicada a todos los efectos en el momento en que se produzca el acceso a su contenido en la dirección electrónica, de modo que pueda comprobarse fehacientemente por el remitente tal acceso; en cualquier caso, si existe constancia de la recepción y transcurren diez días naturales sin que el destinatario acceda a su contenido, se entiende que ha sido rechazada, salvo que de oficio o a instancias del destinatario se compruebe la imposibilidad técnica o material del acceso (art. 15.2).

\section{PROCEDIMIENTOS ADMINISTRATIVOS ELECTRÓNICOS}

Los procedimientos susceptibles de tramitación a través de redes de telecomunicación son los que se relacionan en el Anexo I del Decreto ${ }^{15}$ y los que se establezcan mediante Órdenes de las correspondientes Consejerías ${ }^{16}$. Estas disposiciones han de indicar el plazo máximo para resolver y notificar la reso-

${ }^{13}$ El art. 9.6 especifica que los documentos electrónicos transmitidos a través del Registro telemático serán válidos a todos los efectos legales siempre que quede acreditada su autenticidad, integridad, conservación, identidad del autor y, en su caso, la recepción por el interesado, en la forma prevista para cada caso o tipo de actuación por el art. 12 del Decreto.

${ }^{14}$ El Decreto andaluz es en este punto más flexible que el art. 12 del RD 263/1996, de 16 de febrero, que regula la utilización de técnicas electrónicas, informáticas y telemáticas por la Administración General del Estado.

15 Ello podrá tener lugar desde las cero horas del día siguiente al de la entrada en vigor del Decreto. La normativa y los requisitos técnicos necesarios para la tramitación de los procedimientos se encuentran disponibles en la página web correspondiente de cada Consejería y Organismo Autónomo (Disposición Adicional Cuarta).

${ }^{16}$ Ha de tenerse en cuenta, sin embargo, que expresamente se excluye la aplicación del Decreto a los procedimientos iniciados con anterioridad a su entrada en vigor, así como a los procedimientos iniciados antes de que se incorporen al sistema en virtud de las Ordenes referidas (Disposición Transitoria segunda). 
lución de cada procedimiento y los efectos del silencio, que no podrán ser distintos a los establecidos para su tramitación ordinaria. Además deben especificar en cada caso y en relación con cada uno de los procedimientos contemplados los trámites susceptibles de ser cumplimentados telemáticamente y los que no pueden serlo y la posibilidad, en su caso, de satisfacer el pago de las tasas o exacciones que correspondan por vía electrónica (art. 17.3) ${ }^{17}$.

El artículo 16 aborda la regulación de la iniciación y tramitación de los procedimientos administrativos electrónicos insistiendo en la necesidad de cumplir los requisitos señalados en el art. 12.3 y que la emisión o recepción de documentos se produzca a través del Registro telemático único (apartado primero). El interesado tiene la posibilidad de, una vez iniciado un procedimiento bajo un concreto sistema, practicar actuaciones o trámites a través de otro distinto; en todo caso, en el momento de la aportación de documentos o datos en los Registros deberá indicarse expresamente si la iniciación del procedimiento o alguno de los trámites del mismo se ha efectuado en forma electrónica o telemática (apartado tercero).

\section{CONCLUSIÓN}

Con el Decreto 183/2003 se ha dado un paso ciertamente importante para la implantación de los servicios electrónicos en el ámbito de la Administración de nuestra Comunidad Autónoma, en cumplimiento además del mandato de eficacia que contiene el art. 103 CE. De este modo la Junta de Andalucía avanza hacia la consecución del cuarto nivel de prestación de servicios en este entorno (realización de pagos en línea), situándose de momento en el tercero, esto es, en la posibilidad de interactuar en el seno de los procedimientos administrativos a través de la red, de modo que el ciudadano pueda realizar por vía electrónica y telemática sus trámites y recibir de igual modo la respuesta de la Administración.

El resultado de este esfuerzo es una disposición que ciertamente sigue muy de cerca los precedentes estatales ${ }^{18}$ y cuya efectividad va a depender,

${ }^{17}$ Recuérdese que el art. 38.9 de la Ley 30/1992 dispone que "los registros telemáticos sólo estarán habilitados para la recepción o salida de las solicitudes, escritos y comunicaciones relativas a los procedimientos y trámites ... que se especifiquen en la norma de creación de éste ...”

${ }^{18}$ Aunque la Ley 30/1992 impone en buena medida una normativa común, se ha tenido muy en cuenta la normativa de desarrollo en el ámbito de la Administración General del Estado (vid. por ejemplo la similitud entre los arts. 7 del RD 263/1996 y 12 de este Decreto que comentamos). 
preciso es reconocerlo también, de la facilidad con la que los ciudadanos puedan acceder a las modernas tecnologías.

Sea pues bienvenido este Decreto y esperemos que en particular los principios de simplificación y agilización de trámites alcancen su máxima virtualidad en este nuevo universo digital. 